\title{
EFFECTIVENESS OF INDIRECT SELECTION FOR EGG CHARACTERISTICS USING CERTAIN PLASMA CONSTITUENTS IN THREE LOCAL BREEDS OF CHICKENS
}

\author{
Ensaf A. El Full.
}

Poultry Production Dept., Faculty of Agriculture, Fayoum Univ., Egypt.

\section{ABSTRACT:}

This work aimed to evaluate the effectiveness of the indirect selection by using certain plasma constituents at 8 and 12 weeks of age to achieve an advantageous change of the target productive traits in three local breeds of chickens: Dandarawi (Dand), Fayoumi (Fay) and Golden Montazah (GM). Breed significantly $(\mathrm{P} \leq 0.01)$ affected plasma constituents regardless of age of measurements. Fay had significantly higher PG and PTC than other breeds (200.65 and $108.20 \mathrm{mg} / 100 \mathrm{ml})$ whereas GM had the lowest PTC of $88.91 \mathrm{mg} / 100 \mathrm{ml}$. Dand had the highest PTP being $6.38 \mathrm{mg} / 100 \mathrm{ml}$. Age of measurements significantly affected either PTP or PTC while, its effect on PG was insignificant. Higher PTP at 12 than 8 weeks of age was shown whereas the PTC was significantly higher at 8 weeks of age than 12 weeks of age (6.46 vs 5.69 and 109.79 vs $96.94 \mathrm{mg} / 100 \mathrm{ml}$, Table 1). Significant breed by age of measurement interaction $(\mathrm{P} \leq 0.01)$ effects on $\mathrm{PG}, \mathrm{PTP}$ and PTC were found. There were inconsistent breed differences either in magnitude or direction in the expected correlated responses in egg production-related traits and each of clutch size and pause traits to future selection. Therefore, plasma constituents at 8 and 12 weeks in Dand, GM and Fay can be used as selection criteria to improve their productive performance. So, the more than unity efficiencies indicated indirect selection for target productive traits through selection for plasma constituents at certain studied ages which was more efficient than the direct selection for improving these traits. However, direct selection for productive traits will be the most efficient method for improving these traits if their efficiencies were lower than unity.

Key words: Effectiveness, indirect selection, plasma constituents, egg characteristics, local chickens.

\section{INTRODUCTION}

Selection for improving certain traits sometimes resulted in a correlated response for other unselected traits (secondary characters) which may result from genetic effects which referred mainly to linkage, environmental influences and a combination of both (Falconer, 1989). The indirect selection cannot be expected to be better than direct selection unless the secondary character has a substantially higher heritability and the genetic correlation is high. He illustrated that there are three practical considerations make indirect selection preferable: 1 . If the desired character is difficult to measure with precision, the errors of measurement may so reduce the heritability that indirect selection becomes advantageous. 2. If the desired character is measurable in one sex only, but the secondary character is

Fayoum J. Agric. Res. \& Dev., Vol. 20, No.2, July, 2006 
measurable in both, then a higher intensity of selection will be possible by indirect selection. 3. The desired character may be costly or a very time consuming to measure.

Blood biochemical traits could be important as indicator traits in breeding for high productivity (Obeidah et al., 1978, Abdel Latif, 2001 and Abdel Magid, 2006). Metabolic differences among animals with potential or ongoing production may be useful predictors of genetic merit for economic productive traits (Peterson et al., 1982). The purpose of this study is to evaluate the effectiveness of the indirect selection (through selection for plasma constituents at certain ages) in improving the egg production-related traits and both clutch size and pause traits in three local breeds of chickens after one generation of future selection.

\section{MATERIALS AND METHODS}

This work was carried out at El Azab Poultry Research Center, Animal Production Research Institute, Agricultural Research Center, Ministry of Agriculture, Egypt. A total of 450 females of Dandarawi (Dand), 592 females of Fayoumi (Fay) and 402 females of Golden Montazah (GM) belonging to ten sires and 100 dams were produced in five to seven successive pedigreed hatches, seven days apart, respectively. Management practices were kept uniform as possible throughout the experimental period. Blood samples were collected at 8 and 12 weeks of age. About $3 \mathrm{~cm}^{3}$ of blood from each female were taken from the wing vein in the morning (between 8 and 10 o'clock) before feeding. Blood samples were immediately centrifuged at $3000 \mathrm{rpm}$ for 15 minutes to separate plasma. The prepared plasma samples were stored at $-20^{\circ} \mathrm{C}$ till the time of chemical analyses. Plasma samples were analyzed at the laboratories of Poultry Production Department, Faculty of Agriculture, Fayoum University. Kits of STANBIO Laboratory INC., Texas, USA, were used.

Age at sexual maturity (SM) individually recorded in days for each hen in each breed. Egg number, egg weight and egg mass from sexual maturity up to 90 days of egg production were recorded on each hen $\left(\mathrm{EN}_{90}, \mathrm{EW}_{90}\right.$ and $\left.\mathrm{EM}_{90}\right)$. Two measurements for either clutch or pause traits were considered during the first 90 day of production: the number of eggs per clutch of each hen $\left(\mathrm{CS}_{90}\right)$, the number of clutches $\left(\mathrm{CN}_{90}\right)$ and the average length of pause duration ( $\mathrm{PD}_{90}$, days) were estimated. The following egg quality characteristics were recorded for each hen: Yolk index (YI) Haugh unit (HU), egg shape index (SI) and shell thickness (ST) on the membraneless shells by using Ames shell thickness Gauge to the nearest $\mu \mathrm{m}$.

Plasma glucose (PG, mg/100ml), and total cholesterol (PTC, $\mathrm{mg} / 100 \mathrm{ml}$ ) were quantitatively determined based on an enzymatic-colorimetric technique described by Trinder (1959, 1969), Richmond (1973) and Weissman \& Pileggi (1974). Plasma total protein (PTP, mg/100ml) was calorimetrically determined according to the methods described by Weichselbaum (1946).

Statistical analysis:

Before estimating genetic parameters, data were corrected for hatch effects within each breed. Then data for productive performance traits were analyzed for breed effect as the following model:

$\mathbf{Y}_{\mathrm{ij}}=\boldsymbol{\mu}+\mathbf{B}_{\mathrm{i}}+\mathbf{e}_{\mathrm{ij}}$

Fayoum J. Agric. Res. \& Dev., Vol. 20, No.2, July, 2006 
where:

$\mathbf{Y}_{\mathrm{ij}}$ : expresses the observation of the $\mathrm{ij}^{\text {th }}$ hen, $\boldsymbol{\mu}$ : is the overall mean, $\mathrm{B}_{\mathrm{i}}$ : is the effect of $i^{\text {th }}$ breed $(i=1$ and 3$)$ and $\mathbf{e}_{i j}$ : is the residual error term accounted for the $\mathrm{j}^{\text {th }}$ hen of the $\mathrm{i}^{\text {th }}$ breed. The following model was used for plasma constituent concentrations where breed and age of measurements used as main effects:

\section{where:}

$$
\mathbf{Y}_{\mathrm{ijk}}=\boldsymbol{\mu}+\mathbf{B}_{\mathrm{i}}+\mathbf{A}_{\mathrm{j}}+\mathbf{B} \mathbf{A}_{\mathrm{ij}}+\mathrm{e}_{\mathrm{ijk}}
$$

$\boldsymbol{\mu}$ : Overall mean, $\mathbf{B}_{\mathbf{i}}$ : Breed effect (i: 1 and 3), $\mathbf{A}_{\mathbf{j}}$ : Age of measurement effect (j: 1 and 2), $\mathbf{B} \mathbf{A}_{\mathbf{i j}}$ : Interaction of breed by age of measurement and $\mathbf{e}_{\mathbf{i j k}}$ : Random error term. Means were compared for breed effect and their interactions with age by Duncan's multiple range test (Duncan, 1955) when significant $F$ value were obtained $(\mathrm{P} \leq 0.05)$.

The hierarchical analyses of variance and covariance were done to compute the heritabilities $\left(\mathrm{h}^{2}\right)$ according to Kempthorne (1957) for studied traits using SAS (2000). The following model was fitted, by breed, to each of the traits to calculate the genetic parameters:

$$
\mathbf{Y}_{\mathrm{ijk}}=\boldsymbol{\mu}+\mathbf{S}_{\mathrm{i}}+\mathbf{D}_{\mathrm{ij}}+\mathbf{e}_{\mathrm{ijk}}
$$

where:

$\mathbf{Y}_{\mathrm{ijk}}$ : expresses the observation of the $\mathrm{ijk}^{\text {th }}$ hen, $\boldsymbol{\mu}$ : is the overall mean, $\mathrm{S}_{\mathrm{i}}$ : is the effect of $i^{\text {th }}$ sire, $\mathbf{D}_{i j}$ : is the effect of the $j^{\text {th }}$ dam mated to $i^{\text {th }}$ sire, $\mathbf{e}_{i j k}$ : is the error term accounted for the $\mathrm{k}^{\text {th }}$ hen of the $\mathrm{j}^{\text {th }}$ dam and $\mathrm{i}^{\text {th }}$ sire. The standard errors of heritabilites were obtained according to Swiger $\boldsymbol{e t}$ al (1964). The intensity of selection was determined according to Becker (1985) by assuming a flock contained not less than 400 individuals of each sex and that $20 \%$ of each sex to be selected.

The expected genetic direct response (R) after one generation of individual and family selection were estimated according to Falconer (1989) as follows:

\begin{tabular}{||l|l||}
\hline \hline Method of selection & \multicolumn{1}{|c|}{ Expected direct response (R) } \\
\hline Individual selection & $\mathbf{R}=\mathbf{i} \delta \mathbf{p} \mathbf{h}^{2}$ \\
\hline Family selection & $\mathbf{R}=\mathbf{i} \delta \mathbf{p} \mathbf{h}^{2} *\{1+(\mathbf{n}-1) \mathbf{r}\} /(\mathbf{n})^{1 / 2}\{1+(\mathbf{n}-1) \mathbf{t}\}^{1 / 2}$ \\
\hline
\end{tabular}

where:

i: Intensity of selection (selection differential in standard measure): assumed to be equal for all methods, $\boldsymbol{\delta p}$ : Standard deviation of phenotypic values of individuals, $\mathbf{h}^{\mathbf{2}}$ : Heritability of individual values, $\mathbf{r}$ : Intra-class correlation with full-sib families $\mathbf{r}=\mathbf{1 / 2}$ and with half-sib families $r=1 / 4$, $t$ : Correlation of phenotypic values of members of the families and $n:$ Number of individuals in the families $(n=6)$.

Expected correlated response in the secondary (unselected) trait which called correlated response $\left(\mathbf{C R}_{\mathbf{Y}}\right)$ was determined according to Falconer (1989) as the following:

$$
C R_{\mathbf{Y}}=\mathbf{i} h_{\mathbf{X}} h_{\mathbf{Y}} \mathbf{r}_{\mathbf{g}} \delta \mathbf{p}_{(\mathbf{Y})}
$$

Fayoum J. Agric. Res. \& Dev., Vol. 20, No.2, July, 2006 
where:

$\mathbf{h}_{\mathbf{X}}, \mathbf{h}_{\mathbf{Y}}$ are the square root of heritability of $\mathrm{X}$ and $\mathrm{Y}$ traits, $\mathrm{r}_{\mathrm{g}}$ is the genetic correlation between $\mathrm{X}$ and $\mathrm{y}$ traits, $\boldsymbol{\delta} \mathbf{p}_{(\mathbf{Y})}$ is the phenotypic standard deviation of trait $\mathrm{Y}$, and $\mathrm{i}$ is the selection intensity for trait $\mathrm{X}$.

The expected efficiencies (E) for family selection or individual selection were calculated as follows:

\section{where:}

$$
\mathbf{E}=\mathbf{C R}_{\mathbf{Y}} / \mathbf{D R} \mathbf{R}_{\mathbf{Y}}
$$

$\mathbf{E}$ : is the efficiency of selection, $\mathbf{C R}_{\mathbf{Y}}$ : is the correlated response for $\mathrm{Y}$ trait and $\mathbf{D R}_{\mathbf{Y}}$ : is the direct response for $\mathrm{Y}$ trait.

\section{RESULTS AND DISCUSSION}

Information presented in Table 1 , indicated that breed significantly $(\mathrm{P} \leq$ 0.01 ) affected plasma constituents regardless of age of measurements. Fay had significantly higher PG and PTC than other breeds (200.65 and 108.20 $\mathrm{mg} / 100 \mathrm{ml}$ ) whereas GM had the lowest PTC of $88.91 \mathrm{mg} / 100 \mathrm{ml}$. Dand had the highest PTP being $6.38 \mathrm{mg} / 100 \mathrm{ml}$. Similar results for GM and Dand plasma constituents concentrations were reported by Abdel Latif (2001), Moawad (2002) and Abdel Magid (2006). Age of measurements significantly affected either PTP or PTC however insignificantly affected PG. Higher PTP at 12 than 8 weeks of age was shown whereas the PTC was significantly higher at 8 weeks of age than 12 weeks of age (6.46 vs 5.69 and 109.79 vs 96.94 $\mathrm{mg} / 100 \mathrm{ml}$, Table 1$)$. Significant breed by age of measurement interaction $(\mathrm{P} \leq$ 0.01 ) effects on PG, PTP and PTC were found. The highest PG was 210.22 $\mathrm{mg} / 100 \mathrm{ml}$ for Fay at eight weeks whereas the lowest PG was shown by Dand at eight weeks. Significantly higher estimate of $\mathrm{PTP}_{12}$ was shown by Dand whereas Fay had the lowest PTP at 8 weeks of age $(6.98$ and $5.59 \mathrm{mg} / 100 \mathrm{ml}$, respectively). Fay showed significantly higher $\mathrm{PTC}_{8}$, however the lowest PTC of $83.41 \mathrm{mg} / 100 \mathrm{ml}$ was shown for GM at 12 weeks of age.

The earliest breed attained SM was Dand whereas Fay was the latest breed attaining maturity (170.17 vs 208.55 days, $\mathrm{P} \leq 0.01$ )as shown in Table 2 . Earlier SM was reported by Shebl (1998) and El Full et al. (2005a) for native breeds. Similarly, Dand produced the highest $\mathrm{EN}_{90}$ followed by GM whereas Fay had the lowest estimate ( $\mathrm{P} \leq 0.01,64.16$ and 61.46 vs 23.51 eggs $)$. GM hens laid significantly heavier $\mathrm{EW}_{90}$ and $\mathrm{EM}_{90}$ than other breeds, however, Fay had the lowest $\mathrm{EW}_{90}$ and $\mathrm{EM}_{90}$ (48.22 and $2963 \mathrm{~g}$ vs 39.87 and $937 \mathrm{~g}$ ). The cited estimates of $\mathrm{EN}_{90}$ ranged from 23.1 to 57.04 eggs for most native breeds (El Hossari et al., 1995 and El Full et al.(2005a). However, heavier EW $\mathrm{EW}_{90}$ and $\mathrm{EM}_{90}$ for Dand was reported by El Hammady et al. (1992) and Ragab (1996). Whereas Abdel Galil (1993) cited lower EW of 36.2 for same breed. GM had significantly longer followed by Dand $\mathrm{CS}_{90}$ whereas Fay had shorter $\mathrm{CS}_{90}$ than other breeds ( 3.70 and 3.32 vs 1.53 eggs, $\mathrm{P} \leq 0.01$ ). Higher $\mathrm{CN}_{90}$ was shown for Dand whereas Fay had the lowest $\mathrm{CN}_{90}(\mathrm{P} \leq 0.01,19.22$ and 14.89). The longest $\mathrm{PD}_{90}$ was 9.77 days for Fay, however, Dand had the shortest $\mathrm{PD}_{90}$ than other breeds, this is expected since the former had higher egg number and clutch size than the later. Similar trend for clutch and pause traits was reported by El Full et al.(2005b) and Abdel Magid (2006). Concerning egg quality traits, Fay had significantly higher SI, YI, HU and thicker shells than other breeds. Either Dand or GM had higher variability than Fay for HU and ST

Fayoum J. Agric. Res. \& Dev., Vol. 20, No.2, July, 2006 
traits, since they had larger standard phenotypic deviations for their means (Table 2). These results are in agreement with the findings reported by Abdel Latif (2001) and Abdel Magid (2006).

Table 1. Means $\pm \delta_{P}$ for plasma constituents as affected by breed, age and breed $x$ age of measurements interaction.

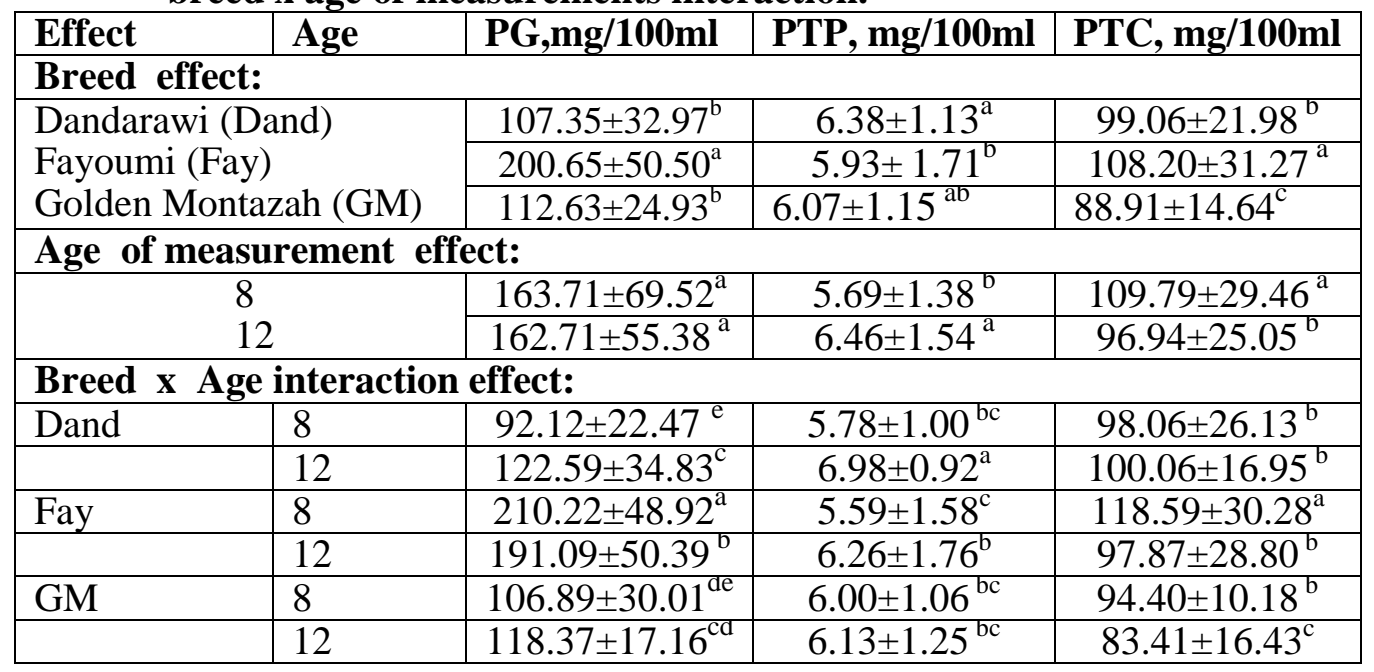

PG : Plasma glucose concentration, PTP: Plasma total protein concentration, PTC: Plasma total cholesterol concentration. Means having different superscripts per each effect within each column are significantly different at $\mathrm{P} \leq 0.01$.

Table 2. Means $\pm \boldsymbol{\delta}_{\mathrm{P}}$ of unselected productive traits in different genetic groups.

\begin{tabular}{|l|c|c|c|}
\hline Trait & Dandarawi & Golden Montazah & Fayoumi \\
\hline Egg production-related traits in the first 90 days of production \\
\hline Sexual maturity, day & $170.17 \pm 4.39^{\mathrm{c}}$ & $175.35 \pm 9.32^{\mathrm{b}}$ & $208.55 \pm 22.30^{\mathrm{a}}$ \\
\hline Egg number, egg & $64.16 \pm 8.21^{\mathrm{a}}$ & $61.46 \pm 8.33^{\mathrm{b}}$ & $23.41 \pm 10.82^{\mathrm{c}}$ \\
\hline Egg weight, g & $39.89 \pm 2.80^{\mathrm{b}}$ & $48.22 \pm 3.36^{\mathrm{a}}$ & $39.87 \pm 3.77^{\mathrm{b}}$ \\
\hline Egg mass, g & $2553 \pm 343^{\mathrm{b}}$ & $2963 \pm 406^{\mathrm{a}}$ & $937 \pm 454^{\mathrm{c}}$ \\
\hline Clutch and Pause traits in the first 90 days of production \\
\hline Clutch size, egg & $3.32 \pm 0.91^{\mathrm{b}}$ & $3.70 \pm 1.24^{\mathrm{a}}$ & $1.53 \pm 0.41^{\mathrm{c}}$ \\
\hline Clutch number & $19.22 \pm 3.97^{\mathrm{a}}$ & $18.08 \pm 4.14^{\mathrm{b}}$ & $14.89 \pm 6.48^{\mathrm{c}}$ \\
\hline Pause duration, day & $1.58 \pm 0.70^{\mathrm{b}}$ & $1.70 \pm 1.62^{\mathrm{b}}$ & $9.77 \pm 15.77^{\mathrm{a}}$ \\
\hline Egg quality traits & $76.57 \pm 3.09^{\mathrm{b}}$ & $75.92 \pm 2.73^{\mathrm{b}}$ & $78.18 \pm 4.31^{\mathrm{a}}$ \\
\hline Shape index, \% & $41.99 \pm 2.46^{\mathrm{b}}$ & $42.13 \pm 1.94^{\mathrm{b}}$ & $50.37 \pm 3.19^{\mathrm{a}}$ \\
\hline Yolk index, \% & $70.69 \pm 7.60^{\mathrm{b}}$ & $72.43 \pm 8.20^{\mathrm{b}}$ & $91.20 \pm 5.61^{\mathrm{a}}$ \\
\hline Haugh unit, \% & $37.40 \pm 3.41^{\mathrm{b}}$ & $36.45 \pm 1.73^{\mathrm{c}}$ & $39.88 \pm 1.09^{\mathrm{a}}$ \\
\hline Shell thickness, mm
\end{tabular}

$\mathrm{a}, \mathrm{b}$ and $\mathrm{c}$ : Means having different superscripts within the same raw are significantly different at $\mathrm{P} \leq 0.05$.

Dand had the highest heritability estimates for $\mathrm{PG}_{8}, \mathrm{PG}_{12}, \mathrm{PTP}_{8}, \mathrm{PTP}_{12}$ and $\mathrm{PTC}_{8}$ than other breeds $(0.73,0.42,0.29,0.57$ and 0.52 , respectively). However, GM had the highest heritability estimate for $\mathrm{PTC}_{12}$ of 0.43 than other breeds (Table 3 ). The cited ranges for plasma constituents in these studied breeds were 0.13 to $0.37,0.12$ to 0.43 and 0.24 to 0.45, respectively by Abdel Latif (2001), El Full (2001), Moawad (2002) and Abdel Magid (2006). Similar trends for PTC and PTP were reported by Obeidah et al. (1978), Rai et al. (1987) and Naryana et al.(1991). Fay had higher heritability estimate for

Fayoum J. Agric. Res. \& Dev., Vol. 20, No.2, July, 2006 
SM than other breeds. However, GM had the highest heritability estimates than other breeds for $\mathrm{EN}_{90}, \mathrm{EW}_{90}, \mathrm{EM}_{90}, \mathrm{CS}_{90}, \mathrm{CN}_{90}$ and $\mathrm{PD}_{90}(0.50,0.77,0.54$, $0.57,0.47$, and 0.81$)$. Higher heritability estimates of SI and YI for Fay were found $(0.78$ and 0.87$)$. Dand had larger heritability estimates than other breeds for both HU and ST (0.99 and 0.59).

Table 3. Heritability estimates $\pm \mathrm{SE}$ of target productive traits and plasma constituents studied in different breeds based on full-sib correlations.

\begin{tabular}{|c|c|c|c|}
\hline Item & Dandarawi & Golden Montazah & Fayoumi \\
\hline \multicolumn{4}{|c|}{ Plasma constituents traits } \\
\hline $\mathrm{PG}_{8}, \mathrm{mg} / 100 \mathrm{ml}$ & $0.73 \pm 0.21$ & $0.13 \pm 0.19$ & $0.54 \pm 0.21$ \\
\hline $\mathrm{PG}_{12}, \mathrm{mg} / 100 \mathrm{ml}$ & $0.42 \pm 0.19$ & $0.20 \pm 0.18$ & $0.23 \pm 0.17$ \\
\hline $\mathrm{PTP}_{8}, \mathrm{mg} / 100 \mathrm{ml}$ & $0.29 \pm 0.23$ & $0.20 \pm 0.19$ & $0.21 \pm 0.10$ \\
\hline $\mathrm{PTP}_{12}, \mathrm{mg} / 100 \mathrm{ml}$ & $0.57 \pm 0.21$ & $0.11 \pm 0.19$ & $0.12 \pm 0.15$ \\
\hline $\mathrm{PTC}_{8}, \mathrm{mg} / 100 \mathrm{ml}$ & $0.52 \pm 0.22$ & $0.23 \pm 0.39$ & $0.11 \pm 0.05$ \\
\hline $\mathrm{PTC}_{12}, \mathrm{mg} / 100 \mathrm{ml}$ & $0.10 \pm 0.25$ & $0.43 \pm 0.17$ & $0.09 \pm 0.14$ \\
\hline \multicolumn{4}{|c|}{ Egg production-related traits } \\
\hline SM, day & $0.24 \pm 0.10$ & $0.12 \pm 0.08$ & $0.27 \pm 0.18$ \\
\hline $\mathrm{EN}_{90}, \mathrm{EGG}$ & $0.11 \pm 0.15$ & $0.50 \pm 0.21$ & $0.45 \pm 0.26$ \\
\hline $\mathrm{EW}_{90}, \mathrm{~g}$ & $0.66 \pm 0.19$ & $0.77 \pm 0.29$ & $0.61 \pm 0.37$ \\
\hline $\mathrm{EM}_{90}, \mathrm{~g}$ & $0.14 \pm 0.16$ & $0.54 \pm 0.21$ & $0.43 \pm 0.34$ \\
\hline \multicolumn{4}{|c|}{ Clutch and Pause traits } \\
\hline $\mathrm{CS}_{90}, \mathrm{egg}$ & $0.26 \pm 0.20$ & $0.57 \pm 0.26$ & $0.31 \pm 0.17$ \\
\hline $\mathrm{CN}_{90}$ & $0.24 \pm 0.22$ & $0.47 \pm 0.24$ & $0.37 \pm 0.26$ \\
\hline $\mathrm{PD}_{90}$, day & $0.62 \pm 0.25$ & $0.81 \pm 0.21$ & $0.45 \pm 0.22$ \\
\hline \multicolumn{4}{|l|}{ Egg quality traits } \\
\hline Shape index, \% & $0.37 \pm 0.12$ & $0.67 \pm 0.19$ & $0.78 \pm 0.41$ \\
\hline Yolk index, \% & $0.82 \pm 0.16$ & $0.29 \pm 0.13$ & $0.87 \pm 0.34$ \\
\hline Haugh unit, \% & $0.99 \pm 0.17$ & $0.46 \pm 0.16$ & $0.75 \pm 0.54$ \\
\hline Shell thickness, $\mu \mathrm{m}$ & $0.59 \pm 0.16$ & $0.20 \pm 0.11$ & $0.32 \pm 0.17$ \\
\hline
\end{tabular}

SM: sexual maturity, EN: Egg number, EW: Egg weight, EM: Egg mass, PG : Plasma glucose concentration, PTP: Plasma total protein concentration, PTC: Plasma total cholesterol concentration, CS: Clutch size, CN:Clutch number and PD: Pause duration.

There were inconsistent breed differences either in magnitude or direction in the expected correlated responses in the target productive performance traits to future selection (Table 4). The direct selection responses to same target traits after only one generation were presented in Table 5. Dividing these correlated responses by the direct responses resulted in the relative efficiency estimation based on individual and full-sib families due to selection for plasma constituent as shown in Tables from 6 to 8. Results of the present study indicated that full-sib family selection had higher direct responses than the individual selection in all studied breeds. The highest responses for selection for sexual maturity, egg number, clutch number, pause duration and yolk index \% were shown in Fay (Table 5). Whereas, GM had the highest response due to full-sib family selection, since Fay had lower heritability for most traits, than other breeds for each of egg weight, egg mass, clutch size and Haugh unit. However, Dand had higher response for shell thickness.

Fayoum J. Agric. Res. \& Dev., Vol. 20, No.2, July, 2006 
EFFECTIVENESS OF INDIRECT SELECTION FOR EGG.

32

Table 4. Correlated responses $(C R)$ in the target traits of different genetic groups due to selection for plasma constituents.

\begin{tabular}{|c|c|c|c|c|c|c|}
\hline \multirow{2}{*}{$\begin{array}{c}\text { Target } \\
\text { trait }\end{array}$} & \multicolumn{6}{|c|}{$C R_{Y}=i h_{X} h_{Y} r_{g} \delta p_{(Y)}$} \\
\hline & \multicolumn{3}{|c|}{$\mathbf{P G}_{8}(\mathbf{X})$} & \multicolumn{3}{|c|}{$\mathbf{P G}_{12}(\mathbf{X})$} \\
\hline $\mathrm{Y}$ & Fay & Dand & GM & Fay & Dand & GM \\
\hline SM,day & -7.868 & -22.140 & 1.316 & 13.771 & 3.304 & -5.432 \\
\hline $\mathrm{EN}_{90}, \mathrm{egg}$ & 7.989 & -2.124 & 2.806 & -6.920 & -0.464 & -3.573 \\
\hline $\mathrm{EW}_{90}, \mathrm{~g}$ & -5.259 & 2.634 & -0.482 & -3.717 & 0.029 & 0.121 \\
\hline $\mathrm{EM}_{90}, \mathrm{~g}$ & 82.695 & -0.723 & 1.181 & -205.882 & -0.229 & -1.505 \\
\hline $\mathrm{CS}_{90}, \mathrm{egg}$ & 0.199 & -0.008 & -0.136 & -0.297 & 0.104 & -0.021 \\
\hline $\mathrm{CN}_{90}$ & -2.839 & 0.168 & 0.483 & -2.170 & -0.334 & 0.119 \\
\hline $\mathrm{PD}_{90}$,day & & 0.009 & -0.005 & & -0.029 & 0.029 \\
\hline SI, \% & -0.469 & -0.412 & 0.230 & 0.946 & 0.365 & 0.342 \\
\hline YI, \% & 0.582 & -0.304 & 0.055 & 0.002 & 0.296 & 0.019 \\
\hline HU, \% & -0.499 & -2.609 & -0.553 & 1.011 & -0.694 & 0.691 \\
\hline \multirow[t]{3}{*}{$\mathrm{ST}, \mathrm{mm}$} & 0.311 & -0.858 & 0.055 & -0.489 & 0.0219 & 0.059 \\
\hline & \multicolumn{3}{|c|}{$\mathrm{PTP}_{8}(\mathrm{X})$} & \multicolumn{3}{|c|}{$\mathrm{PTP}_{12}(\mathrm{X})$} \\
\hline & Fay & Dand & GM & Fay & Dand & GM \\
\hline SM,day & -7.508 & -1.029 & -2.791 & -0.506 & 2.253 & -1.644 \\
\hline $\mathrm{EN}_{90}, \mathrm{egg}$ & -14.063 & -1.405 & 0.319 & 7.463 & -0.620 & -2.299 \\
\hline $\mathrm{EW}_{90}, \mathrm{~g}$ & -1.379 & 1.406 & 2.412 & -3.170 & -0.205 & -0.796 \\
\hline $\mathrm{EM}_{90}, \mathrm{~g}$ & -296.046 & -0.649 & 1.533 & -115.505 & -0.357 & -1.652 \\
\hline $\mathrm{CS}_{90}, \mathrm{egg}$ & -0.119 & 0.367 & 0.021 & -0.068 & 0.114 & 0.202 \\
\hline $\mathrm{CN}_{90}$ & -1.264 & -1.302 & 0.060 & -1.070 & 1.201 & -0.576 \\
\hline $\mathrm{PD}_{90}$, day & 11.836 & -0.009 & -0.017 & 2.615 & -0.079 & 0.013 \\
\hline SI, \% & 1.0257 & -0.073 & -0.324 & 0.239 & 0.088 & 0.105 \\
\hline YI, \% & -0.038 & 0.474 & 0.424 & -1.602 & 0.025 & 0.253 \\
\hline HU, \% & -0.810 & 1.297 & 1.256 & -2.238 & 0.079 & 0.276 \\
\hline \multirow[t]{3}{*}{$\mathrm{ST}, \mathrm{mm}$} & 0.055 & 0.109 & -0.385 & 0.386 & 0.792 & 0.362 \\
\hline & \multicolumn{3}{|c|}{$\mathrm{PTC}_{8}(\mathrm{X})$} & \multicolumn{3}{|c|}{$\mathrm{PTC}_{12}(\mathrm{X})$} \\
\hline & Fay & Dand & GM & Fay & Dand & GM \\
\hline SM,day & 6.241 & 7.166 & -0.850 & -9.782 & -5.828 & -7.016 \\
\hline $\mathrm{EN}_{90}, \mathrm{egg}$ & -10.178 & 0.073 & -2.211 & -5.883 & 0.718 & 4.053 \\
\hline $\mathrm{EW}_{90}, \mathrm{~g}$ & -1.723 & 2.069 & -2.315 & -1.904 & 1.106 & 1.945 \\
\hline $\mathrm{EM}_{90}, \mathrm{~g}$ & -181.086 & 0.654 & -2.241 & -152.545 & 0.582 & 3.373 \\
\hline $\mathrm{CS}_{90}, \mathrm{egg}$ & 0.106 & 0.179 & 0.339 & -0.146 & -0.093 & 0.404 \\
\hline $\mathrm{CN}_{90}$ & 0.604 & -1.337 & -0.837 & -1.324 & 0.594 & -1.151 \\
\hline $\mathrm{PD}_{90}$,day & -0.841 & -0.029 & -0.037 & 6.088 & -0.026 & -0.034 \\
\hline SI, \% & -0.884 & -0.195 & 0.134 & 2.382 & 0.192 & -0.026 \\
\hline YI, \% & 0.262 & 0.444 & -0.316 & 1.087 & -0.042 & 0.072 \\
\hline HU, \% & -1.421 & 0.577 & -0.335 & -1.918 & 0.039 & 0.368 \\
\hline $\mathrm{ST}, \mathrm{mm}$ & 0.349 & -0.024 & -0.221 & -0.233 & 0.279 & 0.014 \\
\hline
\end{tabular}

$\mathrm{h}_{\mathrm{X}}, \mathrm{h}_{\mathrm{Y}}$ are the square root of heritability of $\mathrm{X}$ and $\mathrm{Y}$ traits, $\mathrm{r}_{\mathrm{g}}$ is the genetic correlation between $\mathrm{x}$ and $\mathrm{y}$ traits, $\delta \mathrm{p}_{(\mathrm{Y})}$ is the phenotypic standard deviation of trait, and $\mathrm{i}$ is the selection intensity for trait X, PG: Plasma glucose concentration, Fay: Fayoumi, Dand: Dandarawi, GM: Golden Montazah, SM: Sexual maturity, EN: Egg number, EW: Egg weight, EM: Egg mass, SI: Shape index, YI: Yolk index, HU: Haugh unit, ST: Shell thickness, CS: Clutch size, CN: Clutch number and PD: Pause duration.

Table 5. Direct responses ( $R$ ) in the target productive traits $(Y)$ of different genetic groups due to direct selection for these traits.

Fayoum J. Agric. Res. \& Dev., Vol. 20, No.2, July, 2006 
Ensaf A. El Full.

\begin{tabular}{|c|c|c|c|c|c|c|}
\hline \multirow{3}{*}{ Trait } & \multicolumn{6}{|c|}{$\mathbf{R}=\mathbf{i} \delta \mathbf{p} \mathbf{h}^{2}$} \\
\hline & \multicolumn{2}{|c|}{ Dandarawi } & \multicolumn{2}{|c|}{ Golden Montazah } & \multicolumn{2}{|c|}{ Fayoumi } \\
\hline & RIS & RFS & RIS & RFS & RIS & RFS \\
\hline \multicolumn{7}{|c|}{ Egg production-related traits in the first 90 days of production } \\
\hline Sexual maturity, day & 1.475 & 1.979 & 1.566 & 1.791 & 8.429 & 11.734 \\
\hline Egg number, egg & 1.264 & 1.426 & 5.831 & 10.335 & 6.817 & 11.518 \\
\hline Egg weight, $\mathrm{g}$ & 2.587 & 5.271 & 3.622 & 8.038 & 3.219 & 6.294 \\
\hline Egg mass, $\mathrm{g}$ & 67.228 & 79.122 & 306.936 & 564.34 & 333.508 & 371.05 \\
\hline \multicolumn{7}{|c|}{ Clutch and Pause traits in the first 90 days of production } \\
\hline Clutch size, egg & 0.331 & 0.456 & 0.989 & 1.869 & 0.178 & 0.259 \\
\hline Clutch number & 1.334 & 1.791 & 2.724 & 4.693 & 3.357 & 5.227 \\
\hline Pause duration, day & 0.608 & 1.197 & 1.837 & 4.198 & 9.935 & 16.788 \\
\hline \multicolumn{7}{|l|}{ Egg quality traits } \\
\hline Shape index, \% & 1.601 & 2.436 & 2.561 & 5.528 & 4.707 & 10.522 \\
\hline Yolk index, \% & 2.824 & 6.501 & 0.788 & 1.122 & 3.885 & 9.265 \\
\hline Haugh unit, \% & 10.534 & 27.209 & 5.281 & 257.81 & 5.851 & 12.964 \\
\hline Shell thickness, mm & 2.817 & 5.411 & 0.484 & 0.619 & 0.488 & 0.720 \\
\hline
\end{tabular}

Dand: Dandarawi, GM: Golden Montazah, Fay: Unselected control of Fayoumi, $\delta p$ is the phenotypic standard deviation of trait, and $\mathrm{i}$ is the selection intensity for trait $\mathrm{X}$ and $\mathrm{h}^{2}$ : heritability of the selected trait, RIS: Response due to individual selection and RFS: Response due to full-sib family selection.

Table 6. Expected efficiencies (correlated response/direct response)due to individual or family selection for plasma glucose concentration (PG) in the target traits of different genetic.

\begin{tabular}{|l|c|c|c|c|c|c|}
\hline \multirow{2}{*}{ Trait } & \multicolumn{9}{|c|}{ E=CR $\mathbf{Y} / \mathbf{D R}_{\mathbf{Y}}$} \\
\cline { 2 - 7 } & \multicolumn{2}{|c|}{ Dandarawi } & \multicolumn{2}{c|}{ Golden Montazah } & \multicolumn{2}{c|}{ Fayoumi } \\
\hline $\mathbf{P G}_{\mathbf{8}}(\mathbf{X})$ & $\mathbf{E I S}$ & $\mathbf{E F S}$ & $\mathbf{E I S}$ & $\mathbf{E F S}$ & $\mathbf{E I S}$ & $\mathbf{E F S}$ \\
\hline Sexual maturity, day & -10.897 & -8.122 & 0.822 & 0.718 & -0.933 & -0.671 \\
\hline Egg number, egg & -1.664 & -1.475 & 0.437 & 0.247 & 1.172 & 0.694 \\
\hline Egg weight, g & 0.906 & 0.445 & -0.415 & -0.187 & -1.562 & -0.799 \\
\hline Egg mass, g & -0.877 & -0.745 & 0.285 & 0.155 & 0.248 & 0.223 \\
\hline Clutch size, egg & -0.007 & -0.005 & -0.013 & -0.007 & 1.122 & 0.771 \\
\hline Clutch number & 0.142 & 0.106 & 0.082 & 0.048 & -0.846 & -0.543 \\
\hline Pause duration, day & -0.489 & -0.248 & -0.039 & -0.017 & 1.391 & 0.823 \\
\hline Shape index, \% & -0.490 & -0.322 & 0.111 & 0.052 & -0.099 & -0.045 \\
\hline Yolk index, \% & -0.135 & -0.058 & 0.077 & 0.054 & 0.149 & 0.063 \\
\hline Haugh unit, \% & -0.146 & -0.056 & -0.040 & -0.001 & -0.085 & -0.039 \\
\hline Shell thickness, mm & -0.107 & -0.056 & 0.036 & 0.028 & 0.637 & 0.432 \\
\hline PG 12 (X) & \multicolumn{7}{|c|}{} \\
\hline Sexual maturity, day & 1.613 & 1.202 & -3.366 & -2.944 & -0.060 & -0.043 \\
\hline Egg number, egg & -0.361 & -0.319 & -0.509 & -0.287 & 0.578 & 0.342 \\
\hline Egg weight, g & 0.827 & 0.406 & 0.315 & 0.142 & -0.541 & -0.277 \\
\hline Egg mass, g & -0.275 & -0.234 & -0.361 & -0.196 & -0.346 & -0.312 \\
\hline Clutch size, egg & 0.092 & 0.067 & -0.002 & -0.001 & -0.379 & -0.261 \\
\hline Clutch number & -0.281 & -0.209 & 0.020 & 0.012 & -0.319 & -0.205 \\
\hline Pause duration, day & -1.482 & -0.753 & 0.242 & 0.106 & -0.754 & -0.446 \\
\hline Shape index, \% & 0.431 & 0.283 & 0.164 & 0.076 & 0.051 & 0.023 \\
\hline Yolk index, \% & 0.129 & 0.056 & 0.027 & 0.019 & -0.412 & -0.173 \\
\hline Haugh unit, \% & -0.038 & -0.015 & 0.049 & 0.001 & -0.383 & -0.173 \\
\hline Shell thickness, mm & 0.003 & 0.001 & 0.038 & 0.029 & 0.791 & 0.536 \\
\hline
\end{tabular}

EIS: Efficiency due to individual selection and EFS: Efficiency due to full-sib family selection.

Expected efficiencies that presented in Tables 6,7 and 8 indicated that the more than unity efficiencies indicated that indirect selection for target productive traits through selection for plasma constituents at certain ages

Fayoum J. Agric. Res. \& Dev., Vol. 20, No.2, July, 2006 
studied were more efficient than the direct selection for improving these traits. However, direct selection for productive traits will be the most efficient method for improving these traits if their efficiencies were lower than unity.

Table 7. Expected efficiencies (correlated response/direct response)due to individual or family selection for plasma total protein concentration (PTP) in the target traits of different genetic.

\begin{tabular}{|c|c|c|c|c|c|c|}
\hline \multirow{3}{*}{$\begin{array}{c}\text { Trait } \\
\text { PTP }_{8}(X) \\
\end{array}$} & \multicolumn{6}{|c|}{$\mathrm{E}=\mathrm{CR}_{\mathrm{Y}} / \mathrm{DR}_{\mathrm{Y}}$} \\
\hline & \multicolumn{2}{|c|}{ Dandarawi } & \multicolumn{2}{|c|}{ Golden Montazah } & \multicolumn{2}{|c|}{ Fayoumi } \\
\hline & EIS & EFS & EIS & EFS & EIS & EFS \\
\hline Sexual maturity, day & -0.586 & -0.519 & -1.836 & -1.369 & -0.891 & -.640 \\
\hline Egg number, egg & -0.533 & -0.262 & 0.227 & 0.202 & -0.697 & -0.412 \\
\hline Egg weight, g & 0.019 & 0.017 & 0.801 & 0.393 & -0.428 & -0.219 \\
\hline Egg mass, $g$ & -158.636 & -115.151 & 1.678 & 1.426 & -0.888 & -0.798 \\
\hline Clutch size, egg & 0.080 & 0.059 & 0.006 & 0.004 & -0.666 & -0.458 \\
\hline Clutch number & -2.401 & -1.219 & 0.021 & 0.016 & -0.377 & -0.242 \\
\hline Pause duration, day & -0.175 & -0.115 & -0.438 & -0.223 & 0.881 & 0.522 \\
\hline Shape index, \% & -0.049 & -0.021 & -0.248 & -0.163 & 0.218 & 0.097 \\
\hline Yolk index, \% & 0.056 & 0.022 & 0.164 & 0.071 & -0.009 & -0.004 \\
\hline Haugh unit, \% & 0.269 & 0.139 & 0.045 & 0.018 & -0.139 & -0.063 \\
\hline Shell thickness, mm & 0.038 & 0.020 & -0.042 & -0.022 & 0.113 & 0.077 \\
\hline \multicolumn{7}{|l|}{$\mathrm{PTP}_{12}(\mathrm{X})$} \\
\hline Sexual maturity, day & 1.096 & 0.817 & -1.031 & -0.901 & 1.634 & 1.174 \\
\hline Egg number, egg & -0.480 & -0.426 & -0.359 & -0.203 & -1.015 & -0.601 \\
\hline Egg weight, g & -0.075 & 0.037 & -0.191 & -0.086 & -1.155 & -0.591 \\
\hline Egg mass, $\mathrm{g}$ & -0.481 & -0.408 & -0.378 & -0.206 & -0.617 & -0.555 \\
\hline Clutch size, egg & 0.100 & 0.073 & 0.019 & 0.009 & -1.671 & -1.148 \\
\hline Clutch number & 1.207 & 0.899 & -0.098 & -0.057 & -0.646 & -0.415 \\
\hline Pause duration, day & -3.886 & -1.974 & 0.108 & 0.047 & 0.307 & 0.182 \\
\hline Shape index, \% & 0.104 & 0.068 & 0.051 & 0.024 & 0.201 & 0.089 \\
\hline Yolk index, \% & 0.011 & 0.005 & 0.354 & 0.249 & 0.010 & 0.004 \\
\hline Haugh unit, \% & 0.067 & 0.026 & 0.020 & 0.001 & 0.173 & 0.078 \\
\hline Shell thickness, mm & 0.097 & 0.051 & 0.234 & 0.183 & -1.001 & -0.678 \\
\hline
\end{tabular}

EIS: Efficiency due to individual selection and EFS: Efficiency due to full-sib family selection.

Sexual maturity could be indirectly selected through selection for high $\mathrm{PG}_{8}$ or lower $\mathrm{PG}_{12}$ for Dand or high $\mathrm{PG}_{12}$ for $\mathrm{GM}$ as shown in Table 6. Egg number in Dand could be improved through selection for lower $\mathrm{PG}_{8}$. Conversely, Fay' egg number could be improved through selection for high $\mathrm{PG}_{8}$. Egg weight of the Fay could be increased through selection for lower $\mathrm{PG}_{8}$. On the other hand, high $\mathrm{PG}_{8}$ could be used as a selection criteria in Fay to increase clutch size and resulted in undesirable longer pause duration, therefore selection for lower $\mathrm{PG}_{8}$ could be helpful in shortening this trait. Pause duration could be shortened in Dand through selection for higher $\mathrm{PG}_{12}$ as shown in Table 6.

As shown in Table 7, selection for high $\mathrm{PTP}_{8}$ may result in earliness in sexual maturity and heavier egg mass in the GM. However, selection for lower $\mathrm{PTP}_{8}$ may result in increasing egg mass and clutch number in the Dand. Selection for lower PTP 12 could be used as a selection criteria to earlier sexual maturity in both Dand and Fay. However, GM had inverse situation of that trait. Selection for lower PTP 12 could be used to increase egg number, egg weight, clutch size and shell thickness in the Fay breed. Selection for higher PTP $_{12}$ could be increased clutch number and decreased pause duration as shown in Table 7.

Fayoum J. Agric. Res. \& Dev., Vol. 20, No.2, July, 2006 
Table 8. Expected efficiencies (correlated response/direct response)due to individual or family selection for plasma total protein concentration (PTP) in the target traits of different genetic.

\begin{tabular}{|c|c|c|c|c|c|c|}
\hline \multirow{3}{*}{$\begin{array}{c}\text { Trait } \\
\text { PTC }_{8}(\mathrm{X})\end{array}$} & \multicolumn{6}{|c|}{$E=C R_{Y} / D R_{Y}$} \\
\hline & \multicolumn{2}{|c|}{ Dandarawi } & \multicolumn{2}{|c|}{ Golden Montazah } & \multicolumn{2}{|c|}{ Fayoumi } \\
\hline & EIS & EFS & EIS & EFS & EIS & EFS \\
\hline Sexual maturity, day & 3.0709 & 2.619 & -0.563 & -0.419 & 0.740 & 0.532 \\
\hline Egg number, egg & -1.192 & 0.051 & -1.585 & -1.405 & -0.504 & -.298 \\
\hline Egg weight, $g$ & -0.608 & 0.375 & -0.773 & -0.379 & -0.535 & -0.274 \\
\hline Egg mass, $g$ & -14.065 & 0.672 & -2.466 & -2.095 & -0.543 & -0.488 \\
\hline Clutch size, egg & -0.278 & 0.116 & 0.094 & 0.068 & 0.55 & 0.409 \\
\hline Clutch number & -2.840 & -0.841 & -0.291 & -0.217 & 0.179 & 0.116 \\
\hline Pause duration, day & -1.486 & -0.733 & -0.940 & -0.478 & -0.099 & -0.059 \\
\hline Shape index, \% & -1.972 & -0.152 & 0.104 & 0.068 & -0.188 & -0.84 \\
\hline Yolk index, \% & -2.269 & 0.085 & -0.123 & -0.053 & 0.067 & 0.028 \\
\hline Haugh unit, \% & -10.196 & 0.012 & -0.012 & -0.005 & -0.243 & -0.109 \\
\hline Shell thickness, mm & -2.825 & -0.002 & -0.024 & -0.013 & 0.716 & 0.485 \\
\hline \multicolumn{7}{|l|}{$\mathrm{PTC}_{12}(\mathrm{X})$} \\
\hline Sexual maturity, day & -2.819 & -2.102 & -4.347 & -3.801 & -0.583 & -0.419 \\
\hline Egg number, egg & 0.553 & 0.490 & .626 & 0.353 & -0.863 & -0.511 \\
\hline Egg weight, g & 0.404 & 0.198 & 0.461 & 0.208 & -0.592 & -0.303 \\
\hline Egg mass, $g$ & 0.694 & 0.589 & 0.808 & 0.439 & -0.457 & -0.411 \\
\hline Clutch size, egg & -0.081 & -0.059 & 0.037 & 0.019 & -0.819 & -0.563 \\
\hline Clutch number & 0.495 & 0.369 & -0.195 & -0.113 & -0.395 & -0.253 \\
\hline Pause duration, day & -1.266 & -0.643 & -0.284 & -0.124 & 0.716 & 0.423 \\
\hline Shape index, \% & 0.225 & 0.148 & -0.013 & -0.006 & 0.506 & 0.226 \\
\hline Yolk index, \% & -0.018 & -0.008 & 0.100 & 0.070 & 0.279 & 0.117 \\
\hline Haugh unit, \% & 0.033 & 0.013 & 0.026 & 0.001 & -0.328 & -0.148 \\
\hline Shell thickness, mm & 0.034 & 0.018 & 0.009 & 0.007 & -0.478 & -0.324 \\
\hline
\end{tabular}

EIS: Efficiency due to individual selection and EFS: Efficiency due to full-sib family selection.

Selection for lower $\mathrm{PTC}_{8}$ in Dand as shown in Table 8 can be used to improve Dand' sexual maturity through individual or full-sib family selection favoring the former than the later. As shown in Table 8, selection for lower $\mathrm{PTC}_{8}$ can be applied to increase each of egg number, egg mass, clutch number and all studied quality traits in Dand. Similarly, egg number in the GM can be improved via selection for lower $\mathrm{PTC}_{8}$. It can be seen that selection for lower PTC $_{12}$ could be used to decrease sexual maturity in both Dand and GM based on either individual or full-sib family selection. Also, selection for lower PTC $_{12}$ could be used to shorten pause duration for Dand breed.

Therefore, plasma constituents at 8 and 12 weeks in Dand, GM and Fay can be used as selection criteria to improve their productive performance.

\section{REFERENCES}

Abdel Galil, M. A. (1993). Evaluating the performance of some local breeds of chickens under certain plans of nutrition. Ph. D. Thesis, Fac. Agric., Minia Univ., Egypt. 
Abdel Latif, H.A. (2001). Inheritance of certain plasma constituents and their association with some economic traits in Dandarawi and Golden Montazah hens. M.Sc. Thesis, Fac. Agric., Fayoum, Cairo Univ., Egypt.

Abdel Magid, M.H. (2006). Inheritance of certain plasma constituents and their association with some productive traits in two strains of Fayoumi hens. M.Sc. Thesis, Fac. Agric., Fayoum Univ., Egypt.

Becker, W.A. (1985). Manual of Quantitative Genetics, (4 ${ }^{\text {th }}$ Ed). Academic Enterprises, Pullman, Washington, U.S.A.

Duncan, D.B. (1955). The Multiple Range and Multiple F Tests. Biometrics. 11:1-42.

EI Full, E.A. (2001). Genetic and phenotypic parameters of egg production in relation to certain plasma constituents in Dandarawi and Golden Montazah hens. Egypt. Poult. Sci. 21: 765-793.

El Full, E. A., A.A. Abdel Warith, H.A. Abdel Latif and M.A. Khalifa (2005b). A comparative study on pause and clutch size traits in relation to egg production traits in three local breeds of chickens. Egypt. Poult. Sci. 25: 825-844.

El Full, E.A., H.M. Abdel Wahed, M.M. Namra and A.M.R. Osman and N.A. Hataba (2005a). Results of random sample test for laying performance of nine strains of chickens. Egypt. Poult. Sci. 25: 195-208.

El Hammady, H.Y., H.H. Sharara and T.M. El Sheikh (1992). Effect of feeding regimens and lighting programs on egg production performance of laying hens. Egypt. Poult. Sci. 12: 791-817.

El Hossari, M.A., S.A. Dourgham and A.A. Abdel Warith (1995). The significance of improving the Fayoumi chickens using two Fayoumi lines of the same origin. First Egyptian Hungarian poultry conference 17-19 September. Alexandria Egypt, PP: 218-225.

Falconer, D.S., 1989. Introduction to Quantitative Genetics. $3^{\text {rd }}$ Ed. Longman Group.

Kempthorne, O. (1957). An Introduction to Genetic Statistics. John Wiley and Sons, Inc., New York.

Moawad, N.A. (2002). Inheritance of some plasma constituents and their association with some growth traits in Dandarawi and Golden Montazah chickens. M.Sc. Thesis, Fac. Agric., Fayoum, Cairo Univ., Egypt.

Naryana, R., S. K. Verma and U. D. Gupta (1991). Inheritance of plasma protein, cholesterol and glucose, and their association with egg quality traits in inbred lines of White Leghorn. Ind. J. Anim. Sci. 61: 445-448.

Obeidah, A., H. M. Morad, A. A. Sami and A. Mostageer (1978). Genetic and phenotypic parameters of egg production and some constituents of blood serum in Fayoumi layers. Ann. Génét. Sél. Anim. 10: 47-60.

Peterson, R.G., T.E. Nash and J.A. Shelford (1982). Heritabilities and genetic correlations for serum and production traits of lactating Holsteins. J. Dairy Sci. 65: 1556-1561.

Ragab, M.S. (1996). Effect of energy protein restriction on the performance of Dandarawi and Sinai layers. M. Sc. Thesis, Fac. Agric., Fayoum, Cairo Univ., Egypt.

Rai, D., S.K. Verma, R.B. Prasad and A. Kumar (1987). Genetic association of plasma protein, cholesterol and glucose with egg quality characters in inbred lines of White Leghorn. Ind. J. Anim. Sci. 57: 896-898.

Richmond, W. (1973). Preparation and properties of a cholesterol oxidase from nocardia sp. and its application to the enzymatic assay of total cholesterol in serum. Clin. Chem. 19: 1350.

Fayoum J. Agric. Res. \& Dev., Vol. 20, No.2, July, 2006 
SAS Institute (2000). SAS/Stat User's Guide, Release 8.1, SAS Institute Inc., Cary, NC, USA.

Shebl, M.K. (1998). Estimation of heritability of clutch size using REML and Henderson3 and its relationship with growth and egg production traits in chicken. Egypt. Poult. Sci. 18: 167-182.

Swiger, L.A., W.R., Harvey, D.O. Everson and K.F. Gregeory (1964). The variance of intraclass correlation involving groups with one observation. Biometrics, 20: 818-820.

Trinder, L. (1969). Determination of blood glucose using an oxidization peroxidase system with a non carcinogenic chromogen. Ann. Clin. Biochem. 6: 24-27.

Trinder, P. (1959). Determination of blood glucose using 4-Aminophenazone. J. Clin. Path. 22: 246

Weichselbaum, T.E. (1946). An accurate and rapid method for the determination of proteins in small amounts of blood serum and plasma. American J. Clinical Pathology Technique Section 10: 40-49.

Weissman, N. and V.J. Pileggi (1974). In clinical chemistry. Principals and Technics, $2^{\text {nd }}$ ed., R. H. Henry, Eds, Harper \& Row, Hagerstown, M. D, PP: 646-669.

Fayoum J. Agric. Res. \& Dev., Vol. 20, No.2, July, 2006 